\title{
Análise espacial da leptospirose no Brasil
}

\author{
Spatial analysis of leptospirosis in Brazil
}

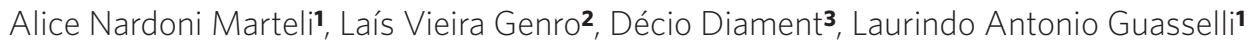

DOI: $10.1590 / 0103-1104202012616$

RESUMO A leptospirose é um problema de saúde pública mundial. No Brasil, ela é uma doença de notificação compulsória e está relacionada a áreas com precárias condições sanitárias. Foi realizado um estudo ecológico retrospectivo para observar a distribuição espacial e temporal de casos confirmados da leptospirose em escala nacional no período entre 2007 e 2017. A espacialização da leptospirose utilizou técnicas de geoprocessamento por análise de padrões pontuais e por mapas de densidade Kernel. Os resultados revelaram que, no período, foram registrados 42.310 casos confirmados de leptospirose no País, com média anual de 3.846 casos e prevalência de 1,9 a cada 100 mil habitantes. As localidades com mais casos prevalentes foram as regiões Sul e Norte. O Sudeste apresentou taxa de prevalência inferior à taxa nacional em virtude da alta densidade demográfica. O método Kernel se mostrou útil na análise global da situação epidemiológica da leptospirose nas regiões do Brasil.

PALAVRAS-CHAVE Epidemiologia. Análise espacial. Banco de dados. Leptospirose.

1 Universidade Federal do Rio Grande do Sul (UFRGS), Programa de Pós-Graduação em Sensoriamento Remoto (PPGSR) - Porto Alegre (RS), Brasil. alicenmart2@gmail.com

2 Universidade Federal do Rio Grande do Sul (UFRGS), Instituto de Geociências (Igeo) - Porto Alegre (RS), Brasil.

3 Instituto de Infectologia Emilio Ribas (IIER) - São Paulo (SP), Brasil.

\begin{abstract}
Leptospirosis is a worldwide public health problem. In Brazil, leptospirosis is a disease of compulsory notification and is related to areas with precarious sanitary conditions. A retrospective ecological study was conducted to observe the spatial and temporal distribution of confirmed cases of leptospirosis on national scale in the period between 2007 and 2017. The spatialization of leptospirosis used geoprocessing techniques by analysis of point patterns and by Kernel density maps. The results showed that within the analyzed period, 42,310 confirmed cases of Leptospirosis were recorded in the country, with an annual average of 3,846 cases and prevalence of 1.9 per 100,000 inhabitants. The regions with the most prevalent cases are the South and the North. The southeast presented a prevalence rate lower than the national rate due to the high demographic density. The Kernel method proved to be useful in the global analysis of the epidemiological situation of leptospirosis in the regions of Brazil.
\end{abstract}

KEYWORDS Epidemiology. Spatial analysis. Database. Leptospirosis. 


\section{Introdução}

As primeiras notificações de casos de leptospirose humana no Brasil ocorreram em 1917, porém, indícios apontam a existência anterior, pois havia confusão dos sintomas com a febre amarela $\mathbf{1}$. A doença já era conhecida desde o século XIX, quando foi estudada por Adolf Weil em humanos em 1886. Stimson, em 1907, deu o nome de Spirocheta interrogans ao microrganismo, depois de observá-lo no fígado de um paciente morto por suspeita de febre amarela. Em 1915, no Japão e depois na Alemanha, comprovou-se que a doença é de natureza contagiosa e microbiana ${ }^{2}$.

Acredita-se que a bactéria chegou ao Brasil com os roedores nos navios negreiros. No Rio de Janeiro, os primeiros surtos relatados aconteceram nos anos 1960 e sempre coincidiram com as tempestades de verão'. Mais recentemente, vem-se confirmando uma média anual de mais de 3.600 casos no País, sendo 375 óbitos, em média, a cada ano ${ }^{3}$.

A leptospirose é uma doença infecciosa febril de início abrupto resultante da infecção por bactérias do gênero Leptospira ${ }^{3}$, que são espiroquetas pertencentes à ordem Spirochaetales e à família Leptospiraceae, as quais compreendem três gêneros: Leptospira, Leptonema e Turneriella ${ }^{4}$. Do gênero Leptospira, são conhecidas dez espécies patogênicas, cinco espécies potencialmente patogênicas (também denominadas intermediárias) e sete espécies saprófitas ${ }^{5,6}$, com mais de 300 sorovares, que são definidos de acordo com a heterogeneidade estrutural no componente carboidrato de seus lipopolissacarídeo ${ }^{7}$. A Leptospira possui a capacidade de viver em ambientes variados, por tempo bem prolongado ${ }^{\mathbf{8}, 9}$.

O Rattus norvegicus é o principal portador da Leptospira interrogans sorovar Icterohaemorraghiae, uma das mais patogênicas para o homem, que é apenas hospedeiro acidental e terminal dentro da cadeia de transmissão ${ }^{\mathbf{3}, 10}$. Em áreas urbanas, os principais reservatórios são os roedores das espécies Rattus norvegicus (ratazana ou rato de esgoto), Rattus rattus (rato de telhado ou rato preto) e Mus musculus (camundongo ou catita) 3,9,11. Esses animais, ao se infectarem, não desenvolvem a doença, sendo apenas portadores. A Leptospira se fixa nos rins desses animais e é eliminada viva no ambiente por meio da urina, contaminando água, solo e alimentos ${ }^{3,10}$.

Algumas ocupações facilitam o contato com a Leptospira, como trabalhadores em atividades de limpeza e desentupimento de esgotos, garis, catadores de recicláveis, agricultores, pescadores e bombeiros ${ }^{2,3}$. Contudo, a maior parte dos casos ainda ocorre em pessoas que habitam ou trabalham em locais com infraestrutura sanitária precária e expostos a urina de roedores ${ }^{3}$. Nas cidades, a principal via de contaminação ocorre por contato com água e lama contaminadas após alagamentos nos períodos de chuvas, 712,13 .

A infecção humana resulta da exposição direta ou indireta à urina de animais infectados, na pele lesada ou íntegra quando imersa em água contaminada ou através de mucosas ${ }^{\mathbf{7} 14}$. A transmissão também é possível pela ingestão de água ou alimentos contaminados. O contágio pessoa a pessoa é raro, mas pode ocorrer pelo contato com urina, sangue, secreções e tecidos de pessoas infectadas ${ }^{3}$.

As formas clínicas da doença podem ser: assintomática, anictérica e ictérica. Na primeira, o paciente é infectado, mas não manifesta sintomas ou sinais. Na forma anictérica, pode desenvolver meningite ou pneumonite intersticial ou hemorrágica. A doença em geral é bifásica, com período inicial de febre, mal-estar e sintomas similares a uma gripe, seguidos de breve período de defervescência e recrudescência da febre e sintomas. A forma ictérica ou Síndrome de Weil cursa com os mesmos sintomas e sinais da forma anictérica, porém com icterícia e falência de diversos sistemas orgânicos, como insuficiência renal aguda, pneumonite hemorrágica, coagulopatia, arritmias cardíacas e choque ${ }^{15,16}$.

No Brasil, não existe vacina humana contra a leptospirose. Para a prevenção da 
doença, além da educação em saúde, são necessárias medidas para redução da população de roedores ${ }^{8}$.

A análise temporal e espacial das doenças é um instrumento na gestão em saúde ${ }^{17}$. Por isso, ferramentas de geoprocessamento, por se constituir em um conjunto de técnicas de coleta, tratamento, manipulação e apresentação de dados espaciais, têm contribuído principalmente em estudos ecológicos das ciências da saúde ${ }^{13}$. Além disso, o uso de Sistema de Informações Geográficas (SIG) em estudos na área da saúde emerge como uma importante ferramenta na inferência espacial em doenças infecciosas. Entretanto, estudos voltados à análise da leptospirose ainda são restritos ${ }^{18-20}$, muitas vezes voltados a dados clínicos 21-25.

Os trabalhos de espacialização da leptospirose no Brasil se limitam a escalas municipais $^{2,9,10,12-14,18,26-31}$, e regionais ${ }^{19,32-35}$. Neste trabalho, o objetivo foi analisar a distribuição espacial e temporal da leptospirose no período entre 2007 e 2017 no Brasil.

\section{Material e métodos}

\section{Área de estudo}

O Brasil possui 5.570 municípios distribuídos em uma área territorial de $8,5 \mathrm{~km}^{2} \mathrm{e}$ uma população estimada em $2018^{36}$ de 208.494 .900 pessoas. A divisão regional do País organiza o espaço brasileiro em cinco regiões (Norte, Nordeste, Sudeste, Sul e Centro-Oeste), objeto deste estudo ecológico retrospectivo de cunho descritivo. Estudos ecológicos em pesquisa epidemiológica avaliam uma população ou um grupo de pessoas que geralmente pertencem a uma área definida ${ }^{17}$.

\section{Fontes de dados}

Os dados de população e leptospirose foram obtidos do Ministério da Saúde pelo Sistema de Informação de Agravos de Notificação (Sinan).
Esse sistema foi desenvolvido entre 1990 e 1993 e tem como objetivo coletar e processar dados sobre agravos de notificação em todo o território nacional das doenças que constam da lista nacional de doenças de notificação compulsória ${ }^{37}$, como, por exemplo, a leptospirose. O Sinan Net disponibiliza esses dados pelo Departamento de Informática do Sistema Único de Saúde ${ }^{38}$.

Os dados de população foram obtidos a partir da estimativa da população residente por município e ano ${ }^{39}$. Os dados de leptospirose foram obtidos dos casos confirmados por município de residência para cada ano entre 2007 e $2017^{40}$. Esses dados foram tabulados e relacionados à malha municipal digital do Instituto Brasileiro de Geografia e Estatística (IBGE) em ambiente SIG. A base cartográfica do IBGE é disponibilizada em formato digital shapefile, tendo como datum o Sistema de Referência Geocêntrico para as Américas (Sirgas 2000) ${ }^{\mathbf{4 1}}$.

A análise comparativa das regiões se deu por meio das taxas de prevalência, que são comumente utilizadas para comparar populações de diferentes tamanhos ${ }^{14}$. A equação para o cálculo da prevalência (P) é11,42,43:

$$
\mathrm{P}=\frac{\left(n^{o}\right. \text { de casos da doença) }}{(\text { população residente })} * 100.000
$$

Para tal, foram considerados o número total de casos confirmados e a população em cada município por ano respectivamente. Após, os dados foram agrupados no QGIS por região geográfica; e em planilha eletrônica da Microsoft Excel $^{\circledR} 2007$, calculadas as taxas de prevalência de forma gráfica.

\section{Análise espacial}

A análise espacial permite identificar padrões de fenômenos geográficos. $\mathrm{Na}$ análise de padrões pontuais (point patterns), a variável de interesse é a própria localização dos eventos. Nesse tipo de análise, o objetivo é saber se os eventos observados ocorreram aleatoriamente 
ou se existe algum padrão sistemático em determinada região ${ }^{17}$.

O Estimador Kernel é uma técnica de interpolação não paramétrica, em que uma distribuição de pontos é transformada em uma superfície de densidade para a identificação visual da ocorrência da concentração do evento, podendo indicar locais de aglomeração ${ }^{44}$. O Kernel possibilita estimar a intensidade, a densidade ou a razão do evento por unidade de área, para análises de cluster ou análises de pontos de calor (hotspot) $\mathbf{4 5 , 4 6}$, e tem sido amplamente aplicada para análise global da situação epidemiológica da leptospirose 2,9,26-28,31.

Os parâmetros considerados são um raio de influência $(t)$ que define a vizinhança circular ao redor da cada ponto (núcleo) da amostra a ser interpolado e uma função de estimação $\mathrm{k}($.) com propriedades de suavização do fenômeno $44,47,48$.

O estimador de densidade Kernel, presente na extensão 'Mapa de Calor' do Sistema de Informação Geográfica QGIS, utiliza cinco funções: Quártica (biponderada), Triangular, Uniforme, Triponderada e Epanechnikov ${ }^{49}$. Nesta análise, foi utilizada a função Triponderada, que resulta em 'pontos quentes mais nítidos'49 e que é dada pela equação ${ }^{\mathbf{4 8}}$ :

$$
\mathrm{K}(t)=35 / 32\left(1-t^{2}\right)^{3},-1 \leq t \leq 1
$$

O raio utilizado (ou largura do Kernel) ${ }^{49}$ foi de $200 \mathrm{~km}$. Para todas as interpolações, a resolução espacial foi padronizada com 2 mil células para o valor de linhas e, as colunas, ajustadas automaticamente pelo software.

Para a estimação da densidade Kernel da taxa de prevalência de leptospirose no Brasil por ano, foram considerados os totais de casos confirmados e a respectiva população por ano. Já para a estimação de densidade de Kernel para leptospirose no Brasil no período de 2007 a 2017, foram considerados o total de casos confirmados de toda a série temporal e a população de 2017. Além disso, foi interpolado o total de casos confirmados por município para efeito comparativo.

\section{Resultados}

Considerando o período de análise entre 2007 e 2017, o gráfico 1 corresponde ao total anual de casos confirmados de leptospirose por região brasileira.

Gráfico 1. Casos confirmados de leptospirose por região brasileira

Centro-Oeste

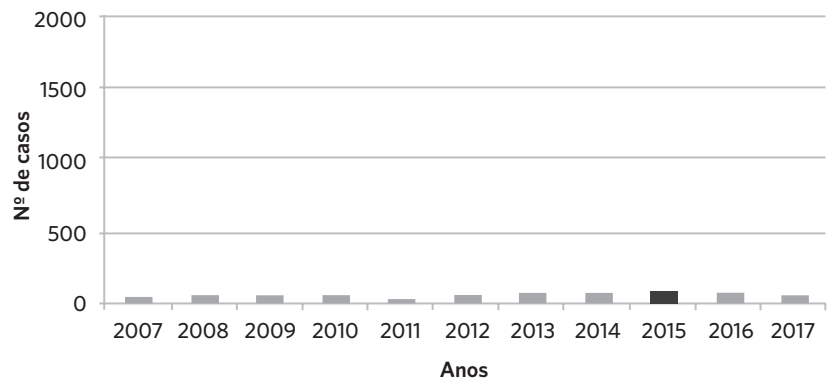

Nordeste

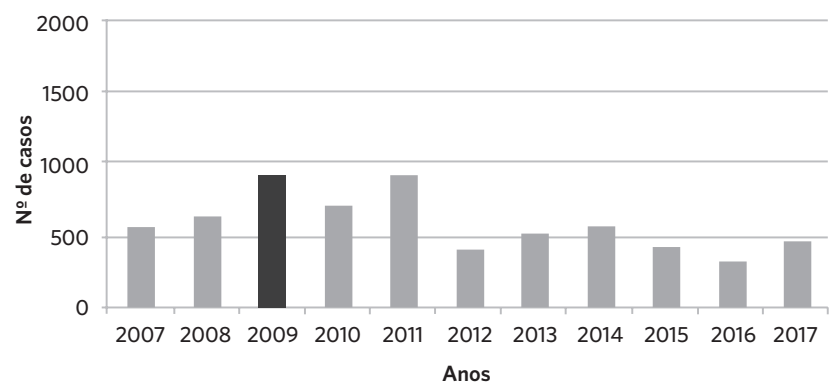


Gráfico 1. (cont.)
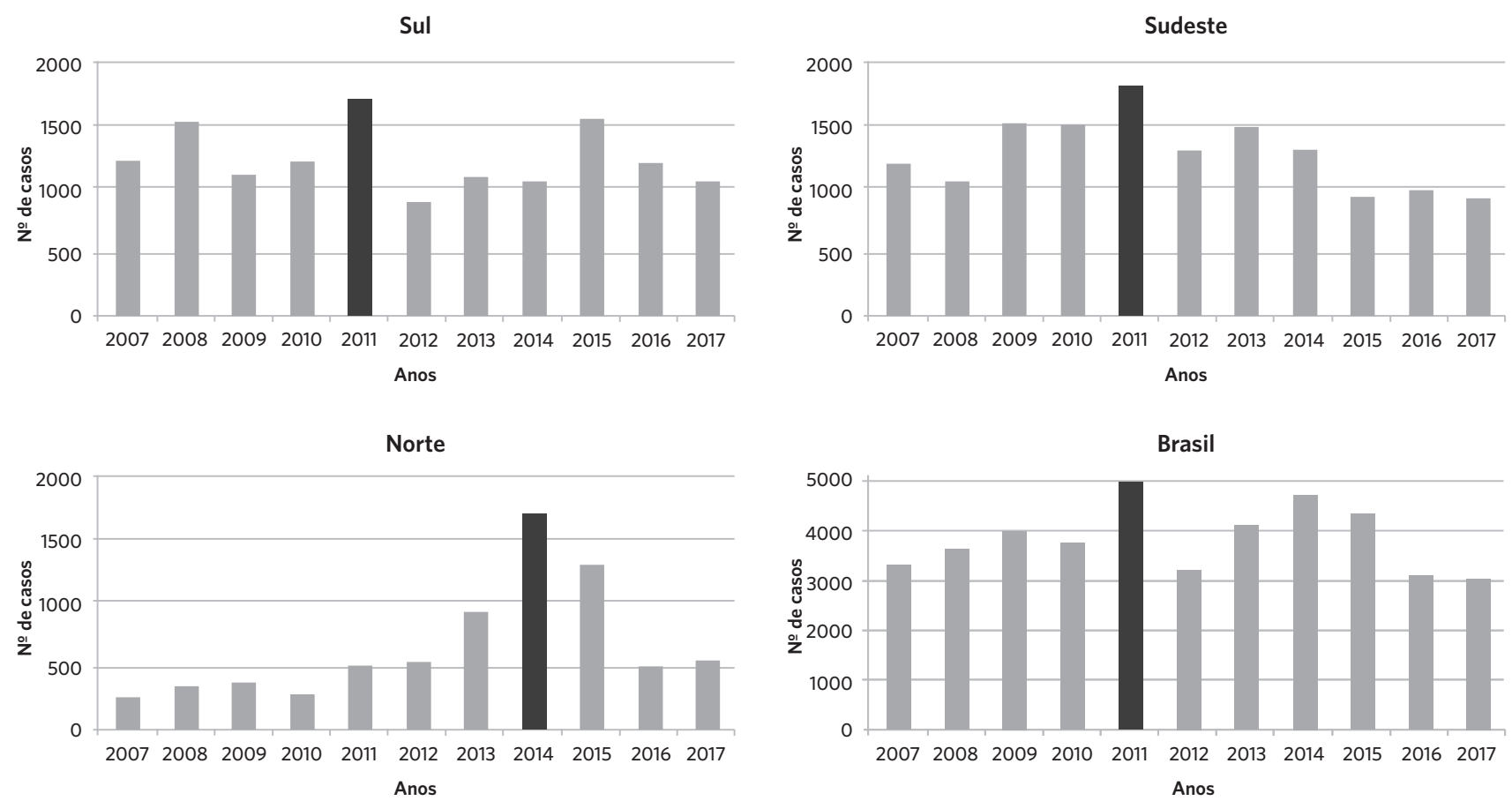

Fonte: Elaboração própria.

Nas regiões Sudeste e Sul, o ano com maior número de casos confirmados foi 2011, com 1.839 e 1.728 respectivamente. No período de 11 anos, a média anual no Sudeste foi de 1.291 casos; e no Sul, de 1.256 casos.

Na região Norte, o ano com maior número de casos foi 2014, com 1.717 casos confirmados; e a média anual do período analisado foi de 654 casos. No Nordeste, 2009 se destacou com 927 casos confirmados, com média anual de 591 casos. Por sua vez, o Centro-Oeste apresentou uma média de 54 casos/ano.

Esse padrão de notificação inferior ao esperado no Centro-Oeste pode significar uma real redução de casos devido à menor densidade populacional, ao atraso no envio das informações, à subnotificação ${ }^{3}$ ou, ainda, possivelmente, devido ao quadro clínico semelhante a outras doenças, gerando subdiagnóstico ${ }^{2,50}$. Além disso, as condições hidrometeorológicas do Centro-Oeste podem ser favoráveis a não disseminação da leptospirose.

A média anual no Brasil de 2007 a 2017 foi de 3.846 casos, o que valida a informação da Secretaria de Vigilância em Saúde ${ }^{\mathbf{3} 43}$ de que, nos últimos dez anos, vem-se confirmando uma média anual de mais de 3.600 casos no País. O total de casos confirmados no período analisado foi de 42.310 .

A distribuição espaçotemporal dos municípios brasileiros que apresentaram pelo menos um caso confirmado de leptospirose entre 2007 e 2017 é representada na figura 1. Dos 5.570 municípios, 2.600 tiveram casos confirmados de leptospirose. 
Figura 1. Distribuição espaçotemporal dos municípios brasileiros com pelo menos um caso confirmado de leptospirose
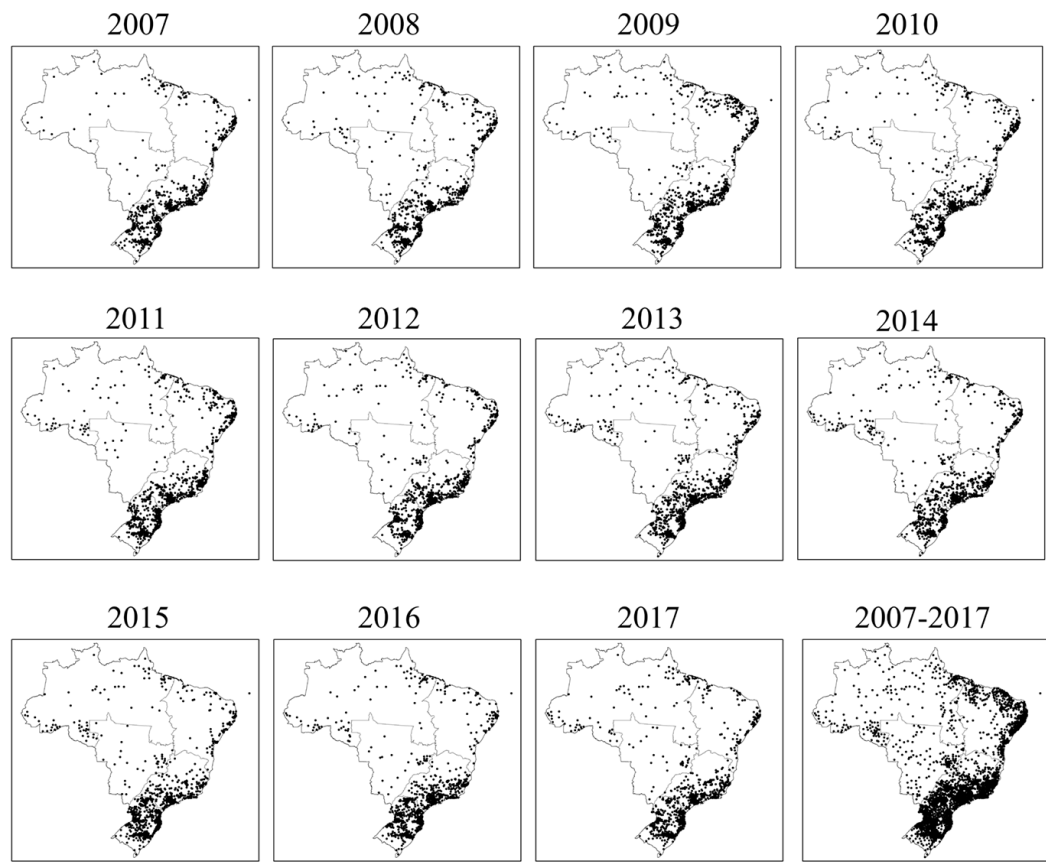

Elaboração: Alice Nardoni Martelii

Datum Horizontal: SIRGAS 2000

Base de Dados: Malhas Digitais IBGE e Sinan Net

- Municípios com casos confirmados de Leptospirose

$\overline{1.000} \quad 2.000 \quad 3.000$

Considerando as diferenças regionais de densidade demográfica no Brasil, além dos totais de casos de leptospirose, foram calculadas as taxas de prevalência anual por região (gráfico 2). Esses dados correspondem à estimativa de risco de contrair leptospirose e à intensidade com que a doença acomete a população.

Gráfico 2. Prevalência anual de casos confirmados de leptospirose

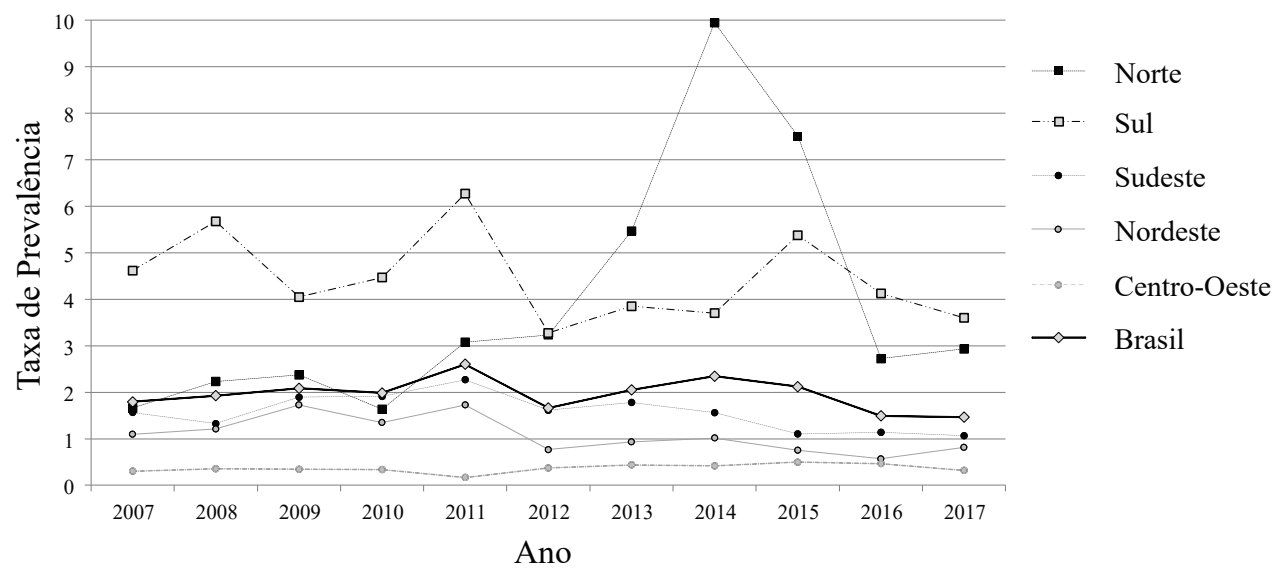


As maiores prevalências estão nas regiões Sul e Norte. Por outro lado, Centro-Oeste, Nordeste e Sudeste apresentaram, nos últimos 11 anos, uma taxa de prevalência menor que a taxa nacional. A prevalência anual no Brasil nesse período foi de 1,9 a cada 100 mil habitantes.
As regiões com maior taxa de prevalência da ocorrência de leptospirose no Brasil por ano são observadas na figura 2. As imagens anuais são representadas com níveis de densidade de acordo a tonalidade. Na faixa mais escura, existe uma concentração elevada da taxa de prevalência de leptospirose.

Figura 2. Estimação da densidade Kernel da taxa de prevalência de leptospirose no Brasil por ano
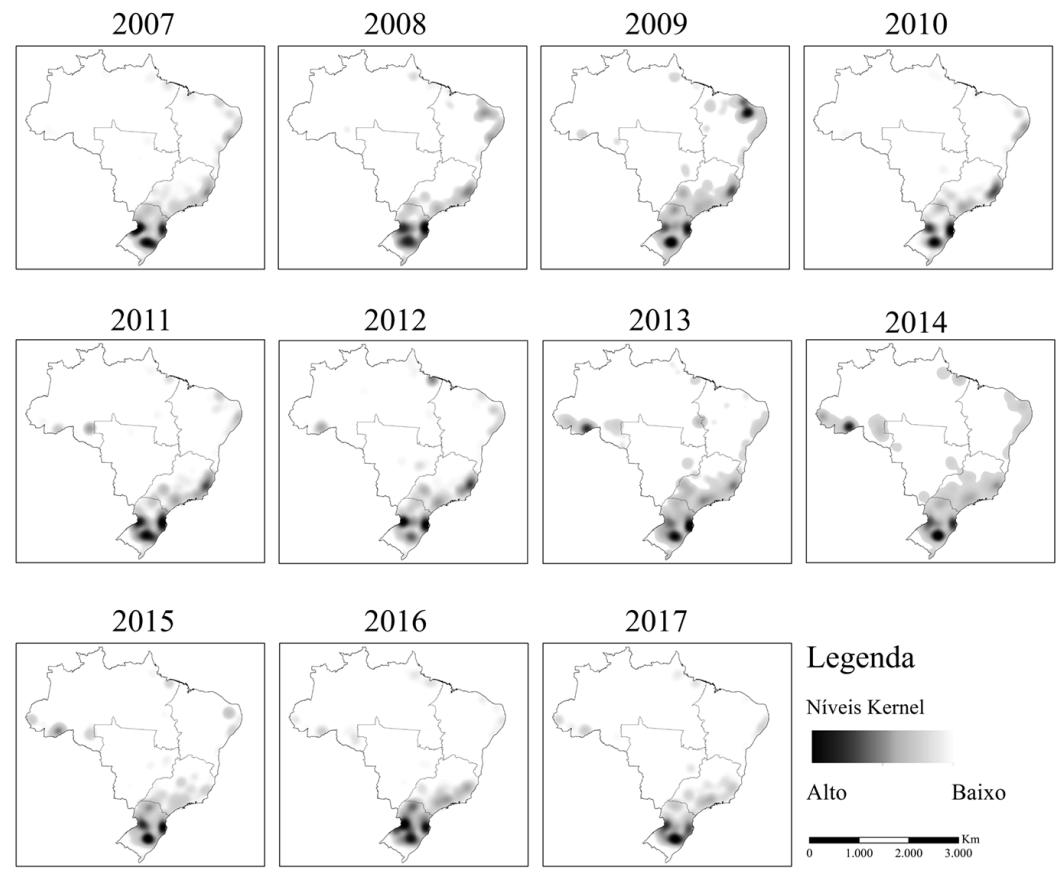

Elaboração: Alice Nardoni Marteli

Datum Horizontal: SIRGAS 2000

Base de Dados: Malhas Digitais IBGE e Sinan Net

LAGAM, CEPSRM - UFRGS, Julho - 2019

Com a estimação de densidade de Kernel para leptospirose no Brasil no período de 2007 a 2017 (figura 3), é possível comparar espacialmente a taxa de prevalência (que considera a população de referência a de 2017) e o total de casos de leptospirose por município (municípios localizados na figura 1, série 2007-2017). 
Figura 3. Estimação de densidade de Kernel para leptospirose no Brasil de 2007 a 2017
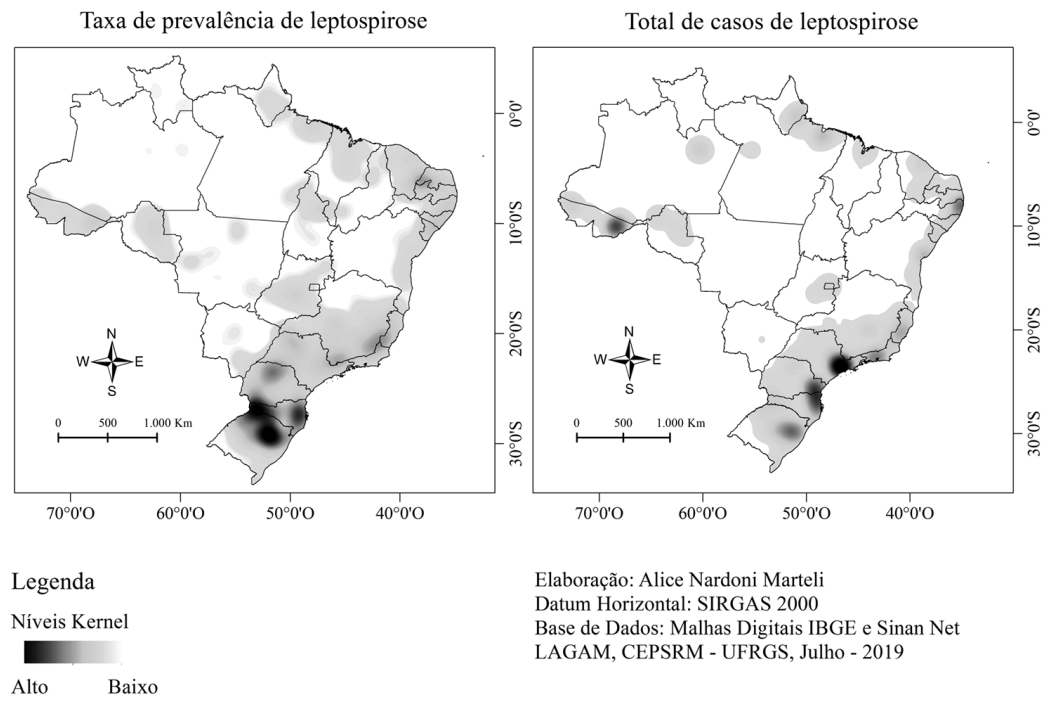

Ao considerar o total de casos na região da capital paulista, o valor dos níveis Kernel é alto, relacionado com a alta quantidade de casos confirmados da doença. Porém, quando considera a população de cada município com caso confirmado (taxa da prevalência), esse valor é menor.

De 2007 a 2017, o município de São Paulo (SP) computou 2.411 casos de leptospirose em uma população de 12.106 .920 habitantes em $2017^{39}$. No mesmo período, o município de Rio Branco (AC) computou 2.598 casos em uma população de 383.443 habitantes em 201739,51. Considerando a escala regional, isso torna o Sul e o Norte as regiões com os maiores casos prevalentes.

Por outro lado, o Sudeste apresentou-se regionalmente com uma taxa de prevalência menor que a taxa nacional (gráfico 2). Esses dados diferem do que afirma o Boletim Epidemiológico que:

[...] no período 2007-2016, as regiões Sudeste e Sul foram responsáveis pelos maiores números de caso por ano, com exceção de 2014, quando

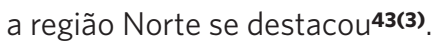

O Boletim Epidemiológico considerou o total de casos, sem considerar a variável população.

\section{Discussão}

A maior parte dos estudos ecológicos em epidemiologia utiliza áreas geográficas como unidade de análise, que representam recortes do espaço geográfico ${ }^{32}$. No Brasil, apesar do avanço com o uso das ferramentas de geoprocessamento para fenômenos relacionados com a área da saúde, a espacialização da leptospirose é limitada em escalas municipais $^{2,9,10,12-14,18,26-31}$ e regionais ${ }^{19,32-35}$.

Trabalhos realizados na cidade do Rio de Janeiro, entre 2000 e $2010^{\circ}$ e entre 2007 e $2012^{12}$, indicaram que áreas com ocorrência de inundações apresentam mais casos de leptospirose. Acrescido a essa relação, Gonçalves ${ }^{13}$ verificou em Belém variáveis demográficas e socioeconômicas como fatores potencializadores dos casos de leptospirose em áreas de baixas condições sanitárias.

Pesquisa realizada no Rio Grande do Sul ${ }^{32}$ sob diferentes recortes ecológicos identificou 
maiores taxas de incidência de leptospirose em áreas litorâneas, de baixa altitude e uso do solo predominantemente agrícola, associada à lavoura irrigada. Embora essas abordagens não sejam comparativas à escala proposta neste trabalho, elas permitem que suas análises se direcionem a instrumentos de gestão em saúde local e regional.

Nesse contexto, Londe et al. ${ }^{30}$ exploraram a temática voltada ao monitoramento e alerta de desastres naturais como apoio à gestão de saúde pública. Segundo os autores, embora as estratégias de redução de risco de desastres tenham sido aprimoradas nos últimos anos, os problemas de saúde geralmente são restritos à resposta de emergência no planejamento de desastres. Por isso, sugerem atribuir ao setor da saúde um papel na prevenção e na preparação para lidar com a redução do risco de desastres com monitoramento integrado dos riscos de desastres e de epidemias.

Apesar das limitações típicas de um estudo ecológico, como a qualidade e a disponibilidade dos dados, este estudo permitiu identificar a leptospirose no Brasil no período de 2007 a 2017 de forma a contemplar todas as regiões que apresentaram casos. Pretende-se associar, em trabalhos futuros, variáveis meteorológicas a essa escala de estudo.

\section{Considerações finais}

A utilização de SIG pode trazer novas abordagens à epidemiologia da leptospirose. O mapeamento dos totais de casos confirmados de leptospirose, sem considerar a população residente, embora muito utilizada nos trabalhos

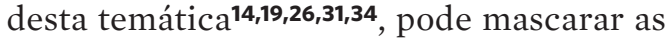
análises quando se planeja alocar recursos de políticas públicas e identificação e intervenção em locais de situações risco à saúde.

$\mathrm{O}$ estimador de densidade Kernel trouxe resultados relevantes para subsidiar a análise espacial da leptospirose no Brasil. A espacialização demonstrou que mapear o total de casos pode ser interessante em termos de atendimento médico. No caso, como observado em São Paulo, o alto nível de densidade Kernel. A análise do total de casos contribui para medidas de atendimento médico; e as áreas com maiores taxas de prevalência permitem a tomada de ações, decisões e intervenções de vigilância em saúde pública sobre a saúde das populações.

A análise de padrões de distribuição de pontos pelo método Kernel se mostrou útil na obtenção de uma análise global da situação epidemiológica da leptospirose no Brasil, como também foi considerada em outras escalas de trabalhos ${ }^{\mathbf{2 6}, 27}$. No entanto, a forte dependência do raio e a excessiva suavização da superfície podem, em alguns casos, esconder variações locais importantes ${ }^{52}$ como foi observado durante a pesquisa.

\section{Colaboradores}

Marteli AN (0000-0002-3467-1190)*, Genro LV (0000-0001-9565-2260)*, Diament D (0000-0003-0147-2061)*, Guasselli LA (00000001-8300-846X)* contribuíram igualmente para a elaboração do manuscrito. ${ }^{*}$ Orcid (Open Researcher and Contributor ID). 


\section{Referências}

1. Oliveira TVS. Fatores socioambientais associados a eventos hidrometeorológicos externos na incidência de leptospirose no município do Rio de Janeiro 1997 a 2009. Rio de Janeiro: ENSP; 2013.

2. Lara JM, Von Zuben A, Costa JV, et al. Leptospirose no município de Campinas, São Paulo, Brasil: 2007 a 2014. Rev Bras Epidemiol [internet]. 2019 [acesso em 2019 abr 1]; (22):2007-14. Disponível em: http://www. scielo.br/scielo.php?script=sci_arttext\&pid=S1415$-790 X 2019000100417 \& \operatorname{lng}=$ pt\&tlng=pt.

3. Brasil. Ministério da Saúde. Guia de Vigilância em Saúde. 3. ed. Brasília, DF; 2019.

4. Levett PN. Systematics of Leptospiraceae. In: Adler B, editor. Leptospira and Leptospirosis [internet]. Berlin: Springer Berlin Heidelberg; 2015. p. 11-20. [acesso em 2019 abr 1]. Disponível em: https://doi. org/10.1007/978-3-662-45059-8_2.

5. Adler B, de la Peña Moctezuma A. Leptospira and leptospirosis. Vet Microbiol [internet]. 2010 [acesso em 2019 jun 24]; 140(3-4):287-96. Disponível em: https://linkinghub.elsevier.com/retrieve/pii/ S0378113509001163.

6. Cerqueira GM, Picardeau M. A century of Leptospira strain typing. Infect Genet Evol [internet]. 2009 [acesso em 2019 jun 24]; 9(5):760-8. Disponível em: https://linkinghub.elsevier.com/retrieve/pii/ S1567134809001403.

7. Picardeau M. Virulence of the zoonotic agent of leptospirosis: Still terra incognita? Nat Rev Microbiol [internet]. 2017; 15(5):297-307. Disponível em: http:// dx.doi.org/10.1038/nrmicro.2017.5.

8. Moura AS, Rocha RL. Endemias e Epidemias: dengue, leischmaniose, febre amarela, influenza, febre maculosa e leptospirose. Belo Horizonte: Nescom; UFMG; 2012. 78 p.

9. Chaiblich JV, Lima MLS, Oliveira RF, et al. Estudo espacial de riscos à leptospirose no município do
Rio de Janeiro (RJ). Saúde debate [internet]. 2017; 41(esp2):225-40. Disponível em: http://www.scielo.br/scielo.php?script=sci_arttext $\&$ pid $=$ S0103$-11042017000600225 \& \operatorname{lng}=$ pt\&tlng=pt.

10. Silva PR. Relações entre a incidência de leptospirose e eventos de inundações urbanas em São José (SC). Florianópolis: Universidade Federal de Santa Catarina; 2013.

11. Fundação Oswaldo Cruz. Instituto de Comunicação e Informação Científica e Tecnológica em Saúde. Taxa de incidência de leptospirose por 100.000. Água Brasil [internet]. [acesso em 2019 jun 24]. Disponível em: https://www.aguabrasil.icict.fiocruz.br/ abrir.php?valor=ASINID55.

12. Guimarães RM, Cruz OG, Parreira VG, et al. Análise temporal da relação entre leptospirose e ocorrência de inundações por chuvas no município do Rio de Janeiro, Brasil, 2007-2012. Ciênc. Saúde Colet. [internet]. 2014 [acesso em 2019 abr 30]; 19(9):368392. Disponível em: https://www.scielo.br/pdf/csc/ v19n9/1413-8123-csc-19-09-3683.pdf.

13. Gonçalves NV, Araujo EN, Sousa Júnior AS, et al. Distribuição espaço-temporal da leptospirose e fatores de risco em Belém, Pará, Brasil. Ciênc. Saúde Colet. [internet]. 2016 [acesso em $2019 \mathrm{fev} 20$ ]; 21(12):394755. Disponível em: https://www.scielo.br/pdf/csc/ v21n12/1413-8123-csc-21-12-3947.pdf.

14. Basso LA, Righi E. Casos de hepatite a e leptospirose no município de Porto Alegre - RS entre os anos de 2007 a 2011. Hygeia. 2015; 11(20):66-84.

15. Lomar AV, Diament D, Torres JR. Leptospirosis in Latin America. Infect Dis Clin North Am. 2000; 14(1):2339.

16. Bharti AR, Nally JE, Ricaldi JN, et al. Leptospirosis: a zoonotic disease of global importance. Lancet Infect Dis [internet]. 2003 [acesso em 2019 maio 28]; 3(12):757-71. Disponível em: https://linkinghub.elsevier.com/retrieve/pii/S1473309903008302. 
17. Medronho RA, Werneck GL, Perez MA. Distribuição das Doenças no Espaço e no Tempo. In: Medronho RA, Bloch KV, Luiz RR, et al, editores. São Paulo: Atheneu; 2009. p. 679.

18. Lira VA, Sá LACM. Aplicação de tecnologias de geoinformação para espacializar os casos de leptospirose. In: Anais $3^{\circ}$ Simpósio Brasileiro de Ciências Geodésicas e Tecnologias da Geoinformação; jul 27-30; Recife. Recife: UFPE; 2010. p. A263.

19. Oliveira MAA, Leal ÉA, Correia MA, et al. Human leptospirosis: occurrence of serovars of Leptospira spp. in the state of Minas Gerais, Brazil, from 2008 to 2012. Brazilian J Microbiol [internet]. 2017 [acesso em 2019 maio 25]; 48(3):483-8. Disponível em: https://linkinghub.elsevier.com/retrieve/pii/S1517838216300545.

20. Gutiérrez JD, Martínez-Vega RA, Botello H, et al. Environmental and socioeconomic determinants of leptospirosis incidence in Colombia. Cad. Saúde Pública [internet]. 2019; [acesso em 2019 maio 25]; 35(3):e00118417. Disponível em: http://www.scielo. br/scielo.php?script=sci_arttext\&pid=S0102-311X2 019000305007\&lng=en\&tlng=em.

21. Daher EF, Lima RS, Silva Júnior GB, et al. Clinical presentation of leptospirosis: a retrospective study of 201 patients in a metropolitan city of Brazil. Brazilian J Infect Dis [internet]. 2010 [acesso em 2019 jul 13]; 14(1):3-10. Disponível em: http://dx.doi.org/10.1016/ S1413-8670(10)70002-7.

22. Blanco RM, Romero EC. Fifteen years of human leptospirosis in São Paulo, Brazil. J Epidemiol Res. 2015; 2(1):56.

23. Daher E, Carvalho GSG, Sousa Soares D, et al. Changing patterns in leptospirosis: a three-decade study in Brazil. Int J Infect Dis [internet]. 2017 [acesso em 2019 maio 7]; 60:4-10. Disponível em: http://dx.doi. org/10.1016/j.ijid.2017.04.023.

24. Sarkar J, Chopra A, Katageri B, et al. Leptospirosis: a re-emerging infection. Asian Pac J Trop Med [internet]. 2012 [acesso em 2019 jul 19]; 5(6):500-2. Disponível em: http://linkinghub.elsevier.com/retrieve/
pii/S1995764512600868.

25. Soares DS, Galdino GS, Rodrigues BC, et al. Arrhythmias in leptospirosis-associated acute kidney injury: a case series. Brazilian J Infect Dis [internet]. 2017 [acesso em 2019 jun 24]; 21(2):209-10. Disponível em: http://dx.doi.org/10.1016/j.bjid.2016.11.010.

26. Nunes FC. Análise espacial da leptospirose na cidade de Salvador-Bahia, no período de 1996-2006. Rio de Janeiro: Fundação Oswaldo Cruz; Escola Nacional de Saúde Pública Sergio Arouca; 2007.

27. Melo CB, Reis RB, Ko AI, et al. Espacialização da leptospirose em Aracaju, Estado de Sergipe, no período de 2001 a 2007. Rev Soc Bras Med Trop [internet]. 2011 [acesso em 2019 jul 25]; 44(4):475-80. Disponível em: http://www.scielo.br/scielo.php?script=sci arttext\&pid=S0037-86822011000400015\&lng=pt\&t lng=pt.

28. Lima RJS, Abreu EMN, Ramos FLP, et al. Análise da distribuição espaço-temporal da leptospirose humana em Belém, Estado do Pará, Brasil. Rev Pan-Amazônica Saúde [internet]. 2012 [acesso em 2019 jul 16]; 3(2):33-40. Disponível em: http://scielo.iec. pa.gov.br/scielo.php?script=sci_arttext\&pid=S2176$-62232012000200005 \& \operatorname{lng}=\mathrm{en} \& \mathrm{nrm}=\mathrm{iso} \& \operatorname{tng}=\mathrm{em}$.

29. Dozsa B, Del Monego MLC, Kummer L. Modelagem Geoestatística da Ocorrência de Casos de Leptospirose e Alagamentos no Município de Curitiba - PR, no Ano de 2014. HOLOS [internet]. 2016 [acesso em 2019 abr 26]; 1:381. Disponível em: http://www2.ifrn. edu.br/ojs/index.php/HOLOS/article/view/3857.

30. Londe LR, Conceição RS, Bernardes T, et al. Flood-related leptospirosis outbreaks in Brazil: perspectives for a joint monitoring by health services and disaster monitoring centers. Nat Hazards [internet]. 2016 [acesso em 2019 jun 24]; 84(2):1419-35. Disponível em: http://link.springer.com/10.1007/s11069016-2493-8.

31. Teixeira KK, Santana RL, Barbosa IR. Associação de variáveis ambientais à ocorrência de leptospirose humana na cidade de Natal-RN: uma análise de distri- 
buição espacial. J Heal Biol Sci [internet]. 2018 Jul 2 [acesso em 2019 maio 26]; 6(3):249. Disponível em: http://periodicos.unichristus.edu.br/index.php/jhbs/ article/view/1766.

32. Barcellos C, Lammerhirt CB, Almeida MAB, et al. Distribuição espacial da leptospirose no Rio Grande do Sul, Brasil: recuperando a ecologia dos estudos ecológicos. Cad. Saúde Pública [internet]. 2003 [acesso em 2019 jun 6]; 19(5):1283-92. Disponível em: http://www. scielo.br/scielo.php?script=sci_arttext\&pid=S0102$-311 X 2003000500007 \& \operatorname{lng}=$ pt\&tlng=pt.

33. Santos LBL, Assis MC, Silva AEP, et al. Sobre risco, ameaça e vulnerabilidade à Leptospirose em situações pós-alagamentos, inundações e enxurradas: reconstruindo o episódio do Vale do Itajaí (2008-2009). In: Anais do Congresso Brasileiro sobre Desastres Naturais; 2012 Maio 14 a 17; Rio Claro. Rio Claro: IGCE/ UNESP; 2012. p. 1-10.

34. Diesel LE, Ometto JPHB. The use of geothechnology for the analysis of prospective risk management: diseases and the occurrence of flooding by rainfall in Santa Catarina state. South of Brazill. Territorium [internet]. 2016 [acesso em 2019 maio 10]; (23):17-22. Disponível em: https://digitalis.uc.pt/handle/10316.2/39726.

35. Buffon EAM, Mendonça FA. Risco socioambiental à leptospirose humana no Aglomerado Urbano Metropolitano de Curitiba, Paraná-Brasil: uma proposta metodológica apoiada em SIG. Ateliê Geográfico [internet]. 2019 [acesso em 2019 jun 2]; 13(1):44-61. Disponível em: https://www.revistas.ufg.br/atelie/ article/view/54065.

36. Instituto Brasileiro de Geografia e Estatística. Cidades 2019 [internet]. Brasília, DF; 2019 [acesso em 2019 maio 20]. Disponível em: https://cidades.ibge. gov.br/brasil/panorama.

37. Braga JU, Werneck GL. Vigilância Epidemiológica. In: Medronho RA, Bloch KV, Luiz RR, et al., editores. 2. ed. São Paulo: Atheneu; 2009. p. 679.

38. Brasil. Ministério da Saúde. Doenças e Agravos de Notificação - De 2007 em diante (SINAN) Departa- mento de Informática do Sistema Único de Saúde DATASUS [internet]. 2019 [acesso em 2019 maio 20]. Disponível em: http://www2.datasus.gov.br/DATASUS/index.php.

39. Brasil. Ministério da Saúde. Sistema de Informação de Agravos de Notificação. População residente por Município e Ano [internet]. 2019 [acesso em 2019 maio 20]. Disponível em: http://www2.datasus.gov. br/DATASUS/index.php?area $=0206 \& i d=6943 \& V O$ bj=http://t.

40. Brasil. Ministério da Saúde. Sistema de Informação de Agravos de Notificação. Leptospirose: Casos confirmados por município de residência de 2007 - 2017 [internet]. 2019 [acesso em 2019 maio 20]. Disponível em: http://www2.datasus.gov.br/DATASUS/index.ph p?area=0203\&id=29892215\&VObj=http://ta\%0Abnet. datasus.gov.br/cgi/deftohtm.exe?sinannet/cnv/lepto.

41. Instituto Brasileiro de Geografia e Estatística. Malha Municipal Digital. Divisão Político-Administrativa (DPA) do Brasil 2018. [internet] Brasília, DF: IBGE [acesso em 2019 maio 20]. Disponível em: https://cidades.ibge.gov.br/brasil/panorama.

42. Wagner MB. Medindo a ocorrência da doença: incidência ou prevalência? J Pediatr (Rio J) [internet]. 1998 [acesso em 2019 jun 24]; 74(2):157-62. Disponível em: https://www.lume.ufrgs.br/bitstream/handle/10183/54350/000164480.pdf.

43. Brasil. Ministério da Saúde. Leptospirose: Situação epidemiológica do Brasil no período de 2007 a 2016. Boletim Epidem. [internet]. 2018 [acesso em 2019 maio 11]; (49):1-7. Disponível em: https:// portalarquivos2.saude.gov.br/images/pdf/2018/ outubro/25/2018-033-Leptospirose-situa----o-epidemiol--gica-do-Brasil-no-per--odo-de-2007-a-2016publica--ao.pdf.

44. Bertolla J. Técnicas de análise de dados distribuídos em áreas. São Paulo: Universidade Estadual Paulista Júlio de Mesquita Filho; 2015.

45. Almeida Júnior HC. Análise da Vulnerabilidade Socioambiental no Município de Vitória-ES, com o apoio 
de um SIG livre. Vitória: Universidade Federal do Espírito Santo; 2017.

46. Minas Gerais. Secretaria de Estado de Meio Ambiente e Desenvolvimento Sustentável. Práticas em geoprocessamento com o Qgis [internet]. 2. ed. Belo Horizonte; Semad; 2019. [acesso em 2019 maio 11]. Disponível em: http://www.meioambiente.mg.gov.br/ images/stories/2019/GESTAO_AMBIENTAL/IDE/ APOSTILA_QGIS_3.4_DGTA-SEMAD-190619.pdf.

47. Cressie NAC. Statistics for Spatial Data [internet]. Hoboken: John Wiley \& Sons, Inc; 1993. [acesso em 2019 maio 11]. Disponível em: http://doi.wiley. com/10.1002/9781119115151.

48. Martinez WL, Martinez AR. Computational Statistics Handbook with MATLAB [internet]. New York: Chapman; Hall/CRC; 2001. [acesso em 2019 maio 11]. Disponível em: https://www.taylorfrancis.com/ books/9781466592742.

49. QGIS Development Team. QGIS User Guide - QGIS Plugins. 2017. [acesso em 2019 jun 14]. Disponível em: https://docs.qgis.org/2.18/en/docs/user_manual/plugins/plugins.html.
50. Rodrigues MC. O círculo vicioso da negligência da leptospirose no Brasil The vicious circle of the leptospirosis negligence in Brazil. Rev Inst Adolfo Lutz. 2017; 76(A6/1729):1-11.

51. Duarte JL, Gitti LL. Incidência da leptospirose em uma capital da Amazônia Ocidental brasileira e sua relação com a variabilidade climática e ambiental, entre os anos de 2008 e 2013. Epid. Serv. Saúde [internet]. 2019 [acesso em 2019 jun 16]; 28(1). Disponível em: http://www.scielo.br/scielo.php?script=sci_ arttext\&pid=S2237-96222019000100305\&lng=pt\&n $\mathrm{rm}=\mathrm{iso}$.

52. Camargo ECG, Fucks SD, Câmara G. Análise espacial de superfícies. In: Fuks SD, Carvalho MS, Câmara G, et al., editores. Análise Espacial de Dados Geográficos [internet]. 2. ed. Planaltina: Ministério da Agricultura; Embrapa; 2004. p. 79-124. Disponível em: http:// livimagens.sct.embrapa.br/amostras/00075490.pdf.

Recebido em 27/09/2019

Aprovado em 23/06/2020

Conflito de interesses: inexistente

Suporte financeiro: Coordenação de Aperfeiçoamento de Pessoal

de Nível Superior - Brasil (Capes) - Código de Financiamento 001 